\title{
SELF-ASSEMBLY OF MICRO PUMPS WITH HIGH UNIFORMITY IN PERFORMANCE
}

\author{
Jiandong Fang, Kerwin Wang, Karl F. Böhringer \\ Department of Electrical Engineering \\ University of Washington \\ Seattle, WA 98195-2500
}

\begin{abstract}
In this paper we report a novel capillary-force-driven selfassembly technique which proceeds in an air environment. We demonstrate this technique for the self-assembly of piezoelectric driving elements (PZT) for diffuser valve micropumps: with the agitation of an orbital shaker, square PZT actuators self-align and mount to the hydrophilic trench binding sites with electric connections by heat curable lubricant oil and conductive physical contact between the PZT actuators and the substrate. 28 pumps are fabricated on a 4-inch bonded pyrex/silicon substrate. The resonance frequencies of all the pumps show high performance uniformity: the average is $80.1 \mathrm{kHz}$ and standard deviation is 2.0 $\mathrm{kHz}$. This technique provides fast, parallel and well-controlled bonding of bulk PZTs for micro pumps with yield ratio up to $100 \%$.
\end{abstract}

\section{INTRODUCTION}

Assembly of microdevices is a key aspect for the development of multi-component integrated micro systems, and capillary-force-driven self-assembly is a prime candidate for the method-of-choice in many situations [1-2]. Previously we reported self-assembly of micro parts in an aqueous environment [3], which limits the application range as follows: 1) parts are soaked in water for a long time, 2) bonding adhesive's volume can not be easily controlled, 3) part tilting is difficult to avoid [4], 4) bonding to hydrophobic binding sites is not strong, 5) electroplating is needed to establish electrical contact. To overcome these disadvantages, this paper reports a novel capillary-force-driven self-assembly technique which proceeds in an air environment. Here we demonstrate this technique for the self-assembly of piezoelectric driving elements for fixed-valve micropumps [5]. The pump consists of a shallow chamber with thin membrane actuated by a PZT actuator, two check valves controlling the flow direction and two channels connecting the check valves to the inlet and outlet holes, respectively. This type of micropump is a good candidate for further miniaturization using self-assembly given the current trend toward control and transport of liquids from microliters to nanoliters [6].

To achieve good performance in piezo driven pumps, the bonding of PZT should have the following properties: 1) adequate stiffness for the pump membrane to bend up and down as the PZT actuator expands and contracts; 2) accurate alignment of the PZT on the pump membrane; 3) controllable bonding thickness without tilt; 4) electrical connections. Usually, highly viscous electrically conductive silver epoxy is used to glue the PZT manually onto these pumps, which is a serial process without good control of process parameters: epoxy thickness can not be controlled easily, epoxy is not uniformly distributed underneath PZT, and alignment errors are difficult to avoid. Using this self-assembly technique, the PZT can be bonded on the pump membrane in a well-controlled parallel process with strong adhesion.

\section{FABRICATION}

Micro pumps are fabricated on a 4" single side polished silicon wafer using two DRIE (System 100 with ICP 380, Oxford Instruments Plasma Technology Ltd, UK) processes and one anodic bonding (Fig. 1). We choose diffuser valves for the micro pumps because they are well understood and reliable [7-9]. AZ4620 (Clariant Inc.) is patterned by photolithography to be the DRIE mask with the following steps: 1) singe the wafer on a 110 ${ }^{\circ} \mathrm{C}$ hotplate for one minute; 2) spincoat primer P20 and AZ4620 at the speed of 2200 RPM for $30 \mathrm{sec}$ in sequence; 3) prebake wafer on a $110{ }^{\circ} \mathrm{C}$ hotplate for 3 minutes; 4) expose with 4" IR contact aligner (AB-M, Inc, USA) UV light for $30 \mathrm{sec}$; 5) develop in solution $A Z 400 \mathrm{~K} / \mathrm{DI}$ water $=1 / 4$ for 2 minutes; 6 ) postbake on a $110{ }^{\circ} \mathrm{C}$ hotplate for 10 minutes. $1^{\text {st }}$ DRIE is for pump chamber ( $5 \mathrm{~mm}$ in diameter, $25 \mu \mathrm{m}$ deep), and $2^{\text {nd }}$ DRIE is for diffuser valves and channels (275 $\mu \mathrm{m}$ deep), Fig. 2 shows the depth uniformity of DRIEs. The shallow and large etched chamber makes the $2^{\text {nd }}$ photolithography of AZ4620 for the channels and valves DRIE feasible, because conformal coating of photoresist on the surface with trenches is difficult. A laser cutter (Model 4410, ESI, USA) is used to drill the inlet and outlet holes. Then a 500 micron-thick 7740 pyrex wafer (Plan Optik, Germany) is anodically bonded to the etched silicon wafer on a $550{ }^{\circ} \mathrm{C}$ hotplate at the voltage of 1200 volts. In order to get thin pump chamber membranes, HF (49\%wt) is used to etch the bonded pyrex wafer down to 230 microns in thickness at the rate of $6.75 \mu \mathrm{m} / \mathrm{min}$. To prepare for the self-assembly of PZT, a layer of Cr/Au (50/1000 $\AA$ ) is deposited by thermal evaporation and patterned by $\mathrm{Cr} / \mathrm{Au}$ etching to form the $3 \mathrm{~mm}$ square binding sites with photoresist AZ1512 as the mask, then AZ1512 is stripped by acetone, finally the exposed pyrex inside the binding sites is etched by HF (49\%wt) to $24 \mu \mathrm{m}$ in depth with the patterned gold as the etching mask. During the above two HF etches, a piece of blue tape is attached to the backside of the pump substrate in order to keep the pump channels and chamber clean from the HF solution.

\section{PZT SELF-ASSEMBLY}

PZT parts are fabricated from a 2.85" square PZT sheet (PSI5H4E, Piezo Systems, Cambridge, MA) with the following steps: 1) AZ1512 is spin coated on the PZT sheet and patterned with the mask protecting the $3 \mathrm{~mm}$ square bonding areas from UV exposure, after developing only the bonding areas are coated with AZ1512;2) $\mathrm{Cr} / \mathrm{Au}(50 / 1000 \AA)$ is deposited on both sides of the PZT sheet by two thermal evaporations; 3 ) the PZT sheet is rinsed in acetone so that AZ1512 and the gold on AZ1512 is removed, the nickel bonding areas are exposed (Fig. 3a); 4) the PZT sheet is diced into $4 \mathrm{~mm}$ square parts; 5) the diced parts are cleaned with acetone, IPA and DI water in sequence inside a sonicator. Finally the cleaned parts are collected to a piece of filter paper and dried by baking on a $100^{\circ} \mathrm{C}$ hotplate for 5 minutes.

Travel support has been generously provided by the Transducers Research Foundation and by the DARPA MEMS and DARPA BioFlips programs. 

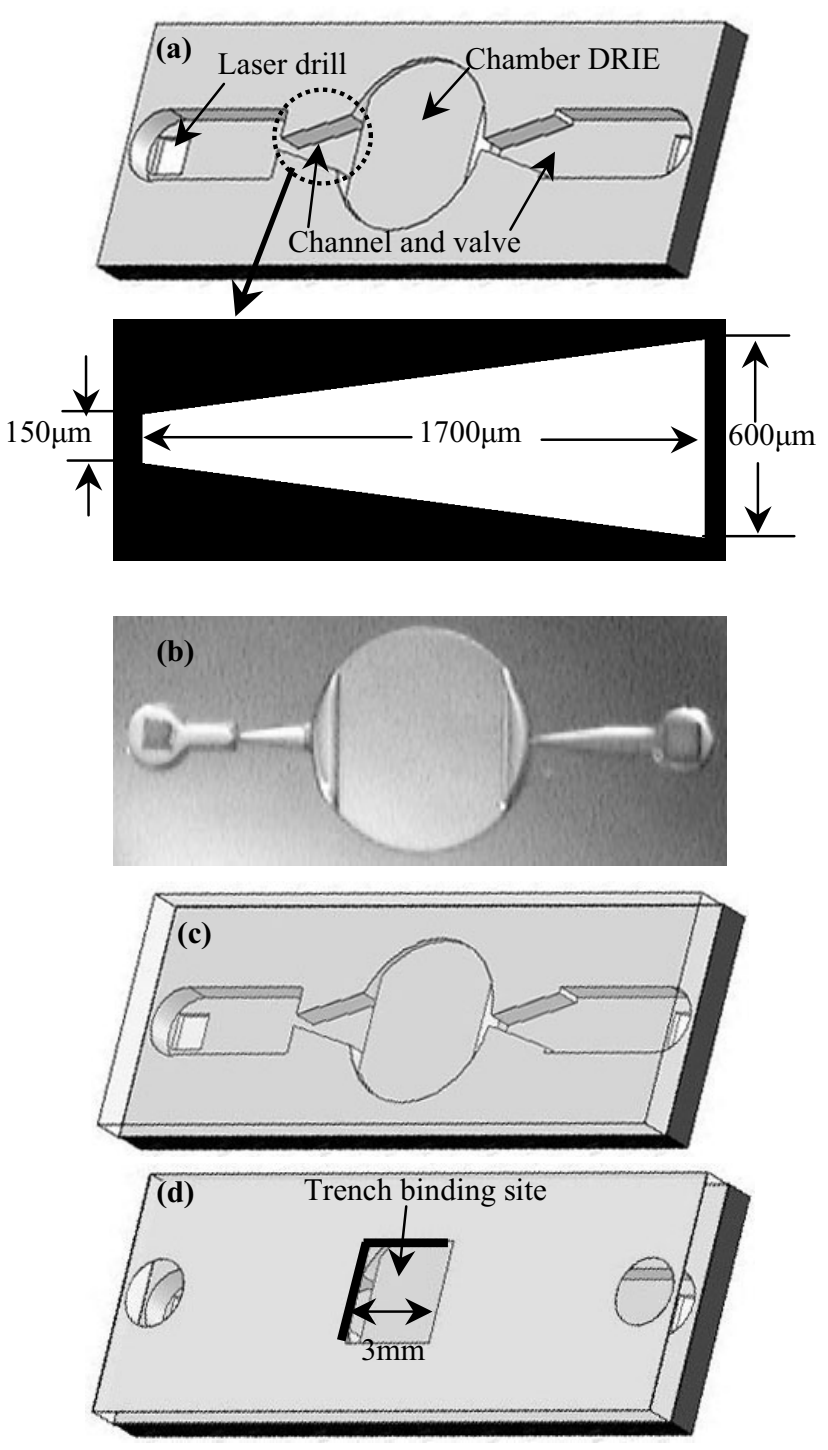

Fig.1. Pump fabrication processes (schematic diagrams not to scale). (a) pump structure after 2 DRIEs and laser drilling; (b) microscope image of the pump structure; (c) anodic boding of pyrex 7740 wafer to the substrate; (d) Cr/Au patterned on the pyrex for the trenched PZT binding sites (two $1 \mathrm{~mm}$ diameter holes on gold are used for alignment of pump with testing stage holes, see Fig. 8).

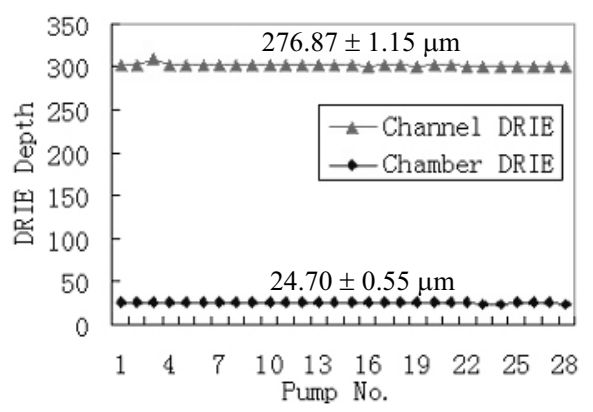

Fig.2. Depth uniformity of two DRIEs: 28 pumps on a single wafer are numbered from top to bottom and from left to right (see Fig. 6). (a)

(c)

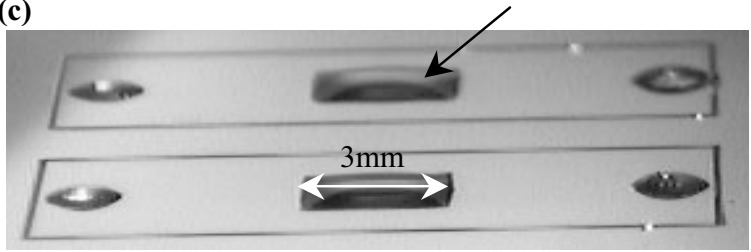

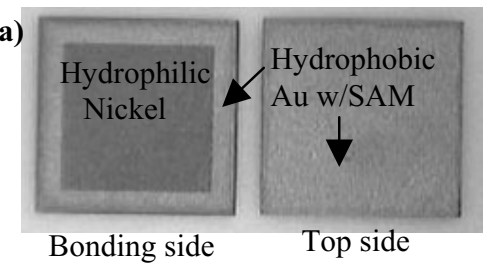

Fig.3. Pump substrate and square PZT part in an air environment. (a) two sides of PZT part; (b) lubricant oil covers only hydrophilic nickel surface of PZT part bonding side; (c) lubricant oil covers only the hydrophilic trench binding sites.

To prepare for self-assembly, the pump substrate and PZT parts are soaked in alkanethiol solution $(48 \mu \mathrm{l}$ 1-dodecanethiol diluted in $100 \mathrm{ml}$ ethanol) for selective adsorption of selfassembled-monolayer (SAM). The gold area on the substrate and parts become hydrophobic after adsorbing SAM from alkanethiol solution by chemical bonding, while the pyrex area on the substrate and nickel area on the PZT parts remain hydrophilic without SAM adsorption after an ethanol rinse.

This self-assembly technique exploits the surface tension force to self-align the PZT parts to the defined $3 \mathrm{~mm} \times 3 \mathrm{~mm} \times 24 \mu \mathrm{m}$ hydrophilic trench binding sites (Fig. 1d). In an air environment heat curable lubricant oil (acrylate-based adhesive) [2] only wets the hydrophilic areas such as the trench binding sites and nickel area on the PZT parts (Fig. 3) and is repelled by hydrophobic SAM coated gold area. Dry PZT parts are introduced manually onto the lubricant oil droplets on the binding sites with the nickel area facing down. The global minimum of the total surface energy can be achieved when the part is aligned with the binding site on the substrate. If the part is not well aligned, the capillary force of the lubricant oil will cause aligning the part with the binding site to minimize the total surface energy. Because the part is larger than the binding site, the part tends to stay in tilt and touches the substrate by its corner or edge, which prevents the capillary force from aligning the part to the binding site. An orbital shaker (Model 51300-00, Cole-Parmer Instrument Company) is used to provide a centrifugal force to the parts, which is uniform everywhere on the shaker stage. The centrifugal force drags and balances the parts on the lubricant oil droplets without touching the substrate so that the parts can self-align with the binding sites by the capillary force to the global minimum surface energy state (Fig. 4).

Finally a 4" pyrex wafer is used to squeeze out the excessive lubricant underneath aligned PZT parts (the top side of the PZT is a hydrophobic SAM coated gold surface, which can prevent the squeezed lubricant from climbing along the hydrophilic sidewalls of the PZT to the top side, otherwise, the lubricant could bond the pressing pyrex wafer with the PZT top side), and electrical connections between the PZTs with surface roughness of about $1 \mu \mathrm{m}$ and the substrate are established (Fig. 5). The lubricant oil is polymerized on an $85^{\circ} \mathrm{C}$ hotplate for about half an hour. The top electrode of the PZT is connected by wire bonding (Fig. 5c). 
(a)
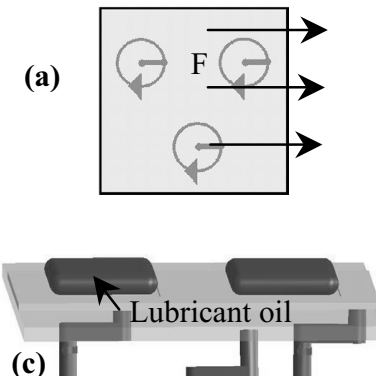

(c)
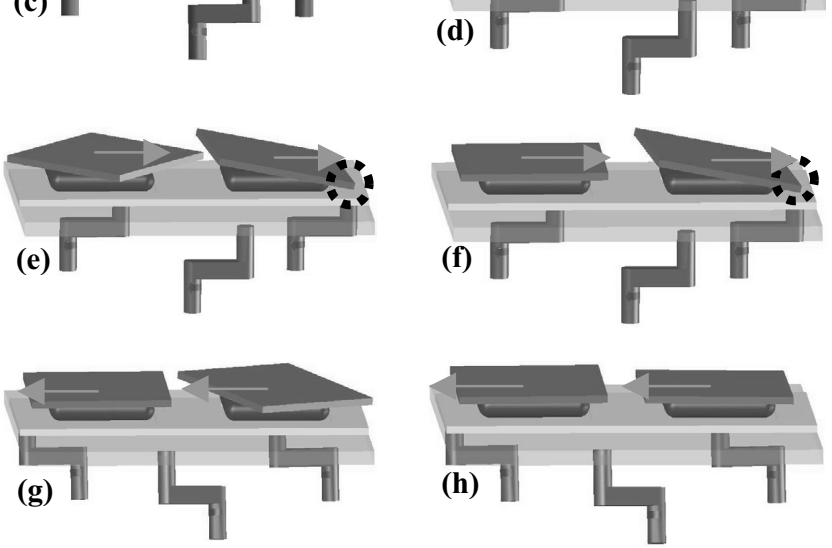

Fig.4. Schematic diagrams for the mechanism of orbital shaker assisted self-alignment. (a) top view of orbital shaker at the phase when the centrifugal force is in the direction to the right; (b) a substrate with two hydrophilic binding sites is attached to the orbital shaker platform; (c) lubricant oil covers only hydrophilic binding sites; (d) two PZT parts are attracted to lubricant oil droplets and stay in a tilt state (one corner is touching the substrate); (e) centrifugal force to the right drags and balances part I on the lubricant oil droplet; (f) part I self-aligns with the binding site; (g) the centrifugal force to the left makes part II flat; (h) part II self-aligns with the binding site.

(a)

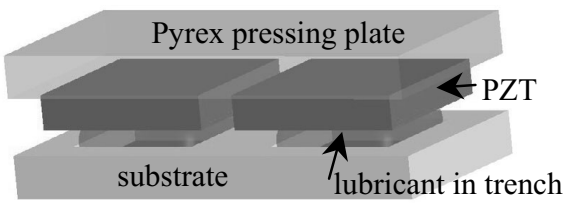

(b)

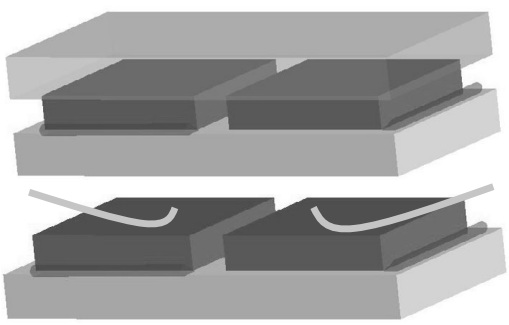

Fig.5. A 500 micron thick pyrex wafer is used to squeeze out excessive lubricant. (a) pyrex wafer is applied on the substrate in parallel; (b) lubricant is squeezed out and PZTs touch the substrate; (c) wires bonded to the PZT top surface for testing use.

\section{PUMPING TEST}

To demonstrate the performance uniformity of all the 28 pumps on a single wafer (Fig. 6), a laser vibrometer is used to obtain the resonance frequency without any fluid load (Fig. 7). Pump \# 9 is chosen for an ethanol pumping test on the reusable testing stage (Fig. 8). During the pumping test, the inlet and outlet Teflon tubes (both tubes are about $15 \mathrm{~cm}$ long) are mounted to a level workstation surface, and the time is measured for the water column head to move through a selected region $(1 \mathrm{~cm} \mathrm{long})$ in the outlet tube. Because the tube fluidic resistance increases with the length of the tube [10], the water column head speed will drop as it moves towards the end of the outlet tube if the driving signal on the PZT does not change. To keep consistency between different frequencies, the water column head is reset to the same initial position before applying a new driving frequency. The result shows the first resonance frequency is about $7.1 \mathrm{kHz}$, and pumping rate at this resonance frequency is about $0.386 \mu \mathrm{l} / \mathrm{sec}$ when the applied voltage is 90 volts (p-p) (Fig. 9).

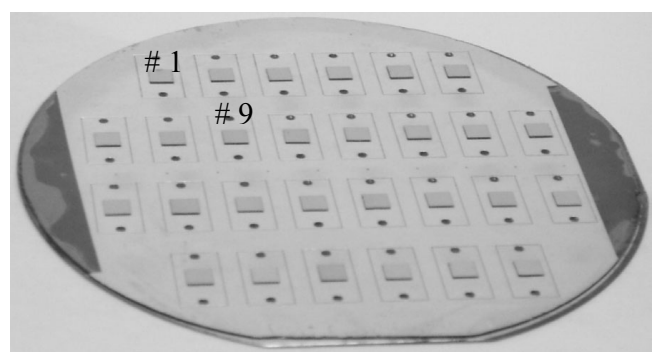

Fig.6. 28 PZTs assembled on pump chamber membranes on a 4inch wafer by orbital shaker assisted self-assembly method. Yield ratio is $100 \%$.

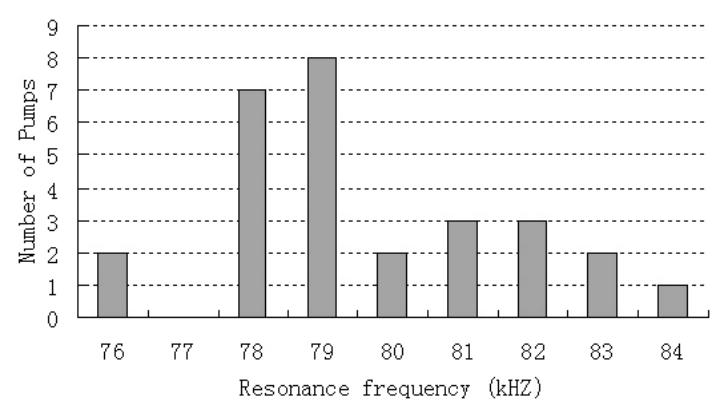

Fig.7. Distribution of resonance frequency of 28 pumps on a single wafer. The average is $80.1 \mathrm{kHz}$, and standard deviation is $2.0 \mathrm{kHz}$. Here the pump wafer is not bonded to the testing stage.

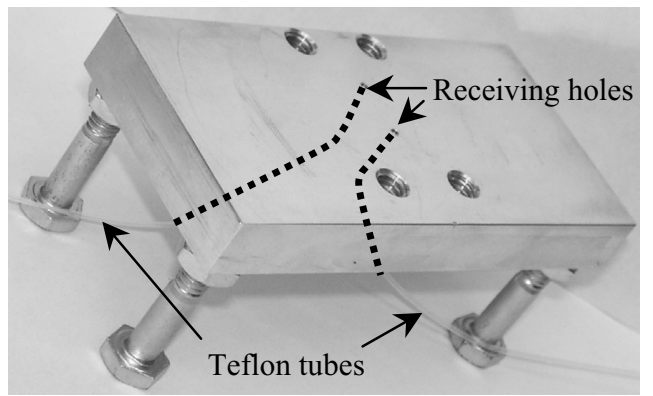

Fig.8. A reusable pump testing stage. Pump wafer was bonded to the stage using silicone rubber, which provides strong bonding and can be de-bonded without any residue by applying an appropriate shear force on the wafer.

\section{ADHESION TEST}

A de-bonding test was performed to compare the adhesion strength of the bonding between in-air assembly method and inwater assembly method. To measure the de-bonding force, a digital 
scale is used (Fig. 10): the bonded stack is held vertically with the PZT resting on a pushing tip that is attached to the digital scale. Then the digital scale reading is zeroed out, and a force is applied to the top of the stack until the PZT is de-bonded or damaged. The final scale reading (in kilograms) multiplied by $\mathrm{g}=9.81 \mathrm{~N} / \mathrm{kg}$ is the de-bonding force. The result shows that bonding to a hydrophilic site without SAM in air is much stronger than bonding

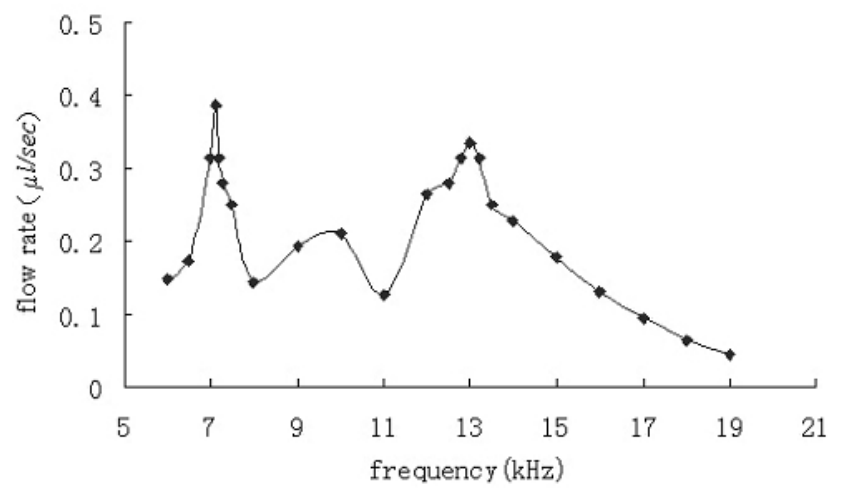

Fig.9. Flow rate vs. frequency of pumping ethanol. The base resonance frequency is about $7.1 \mathrm{kHz}$. Here pump is bonded to the testing stage.

to a hydrophobic site with SAM in water: the maximum shear stresses for in-air and in-water self assembled PZTs are greater than $2.22 \times 10^{6} \mathrm{~Pa}$ (PZT edge damaged by pushing tip at this stress) and $8.89 \times 10^{5} \mathrm{~Pa}$, respectively.

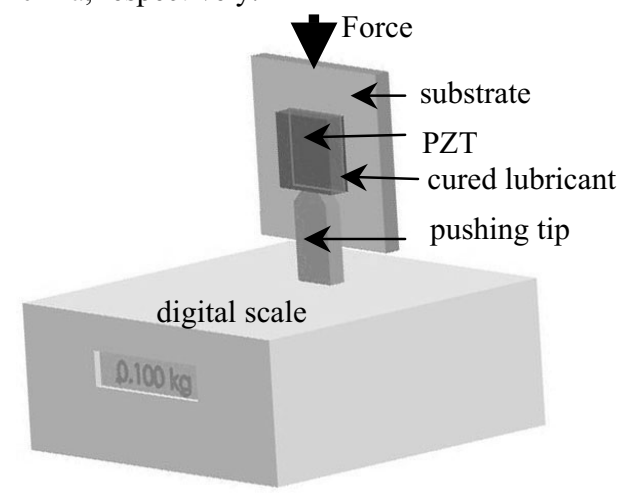

Fig.10. Bonding strength test: 1) $3 \mathrm{~mm}$ square PZT bonded by inwater assembly can be de-bonded by the shear force of about 8 Newtons and the de-bonding is at the interface between lubricant and substrate, which means the SAM on the binding site weakens the bonding; 2) the pump PZT bonded by in-air assembly still stays bonded at the force of 20 Newtons, when the PZT edge is damaged by the pushing tip.

\section{CONCLUSIONS}

An orbital shaker assisted self-assembly technique in air is used to construct fixed valve micro pumps, and all the pumps work with high uniformity. This technique demonstrates many benefits of this method: precise placement, tilt free, strong bonding, welldefined bonding layer thickness by $\mathrm{HF}$ etching, and contact electrical connections by pressing. With batch process capabilities, this self-assembly technique supports the construction of more versatile and miniaturized micro systems.

\section{ACKNOWLEDGEMENTS}

This work is supported by NIH Center of Excellence in Genomic Science and Technology grant 1-P50-HG002360-01. The authors would like to thank Fred Forster and Yael Hanein for inspiring discussions, Brian Williams and Chris Morris for helping with the pump design and pumping test, Tai-Chang Chen for helping with laser cutting, and group members for helpful discussions.

\section{REFERENCES}

[1] J. Lienemann, A. Greiner, J. G. Korvink, X. Xiong, Y. Hanein, K. F. Böhringer, "Modelling, Simulation and Experimentation of a Promising New Packaging Technology - Parallel Fluidic SelfAssembly of Micro Devices." Sensors Update 13(1):3-43, December 2003.

[2] U. Srinivasan, M. H. Helmbrecht, C. Rembe, R. S. Muller, R. T. Howe, "Fluidic Self-Assembly of Micromirrors onto Microactuators Using Capillary Forces," Journal on Selected Topics in Quantum Electronics, 8(1), pp. 4-11, 2002.

[3] X. Xiong, Y. Hanein, J. Fang, Y. Wang, W. Wang, D. T Schwartz, K. F. Böhringer, "Controlled Multi-Batch SelfAssembly of Micro Devices." IEEE Journal of Microelectromechanical Systems 12(2), pp.117-127, 2003.

[4] K. L. Scott, R. T. Howe, C. J. Radke, "Model for Micropart Planarization in Capillary-based Microassembly," Transducers 2003, pp.1319-1322.

[5] F. K. Forster, R. L. Bardell, M. A. Afromowitz, N. R. Sharma, A. Blanchard, Proceedings of the ASME Fluids Engineering Division, ASME International Mechanical Engineering Congress and Exposition (San Francisco), vol. 234 , pp. 39-44. 1995.

[6] B. E. Williams and F. K. Forster. "Micropump design of optimum pressure/flow characteristics". Micro-Electro-Mechanical Systems (MEMS), vol. MEMS-3 ASME International Mechanical Engineering Congress and Exposition (New York), 2001.

[7] K. Yang, I. Chen, B. Shew, C. Wang, "Investigation of the flow characteristics within a micronozzle / diffuser". Journal of Micromechanics and Microengineering, Vol.14, pp.26-31, 2004.

[8] A. Olsson, G. Stemme, E. Stemme, "A numerical design study of the valveless diffuser pump using a lumpedmass model", $J$. Micromech. Microeng. 9, 34-44, 1999.

[9] M. Heschel, M. Muellenborn, S. Bouwstra, "Fabrication and characterization of truly 3-D diffuser/nozzle microstructures in silicon", IEEE J. Microelectromech. Systems, Vol. 6, No. 1, 41-47, March 1997.

[10] C. J. Morris, F. K. Forster, "Low-order modeling of resonance for fixed-valve micropumps based on first principles", IEEE Journal of Microelectromechanical Systems 12(3), pp.325-334, 2003. 